\title{
NICOLÁS DE CUSA: PERSPECTIVAS ÉTICAS A PARTIR DE SU CONCEPCIÓN DEL INDIVIDUO Y DE LA VISIÓN DE DIOS
}

Jorge Mario Machetta*

SÍNTESIS - Alguna consideración reducida de la filosofia medieval considera la síntesis lograda en el siglo XIII como el modelo exclusivo y acabado, de modo que, de ahí en más, los pensadores posteriores ingresen en una pendiente de decadencia que habrá de culminar com la ruptura proclamada por el pensamiento moderno. Pero la historiografía modema nos permite evaluar mejor la diversidad de las propuestas que jalonan los siglos XIV y XV. Uno de estos motivos nos lo proporciona el pensamiento de Nicolás de Cusa (1401-1464) pues en él se evidencia cómo la historia viviente incorpora el pasado y se proyecta hacia el futuro interpretando com oniginalidad su tiempo. La presente investigación pretende incursionar en uno de los aspectos quizá menos explorados del pensar cusano: su perspectiva ética. Nicolás de Cusa no escribió ningún tratado específico. Sin embargo nos legó un enfoque que consideramos muy rico. El punto de partida nace com su concepción de la singularidad. El individuo no es una mónada aislada y desconexa del universo. Para Nicolás de Cusa en cada realidad se da lo máximo "contracto". Se trata de una densa expresión que expressa la presencia de lo infinito en lo finito. Cada entidad, que participa del universo es también irrepetible e insustituible. Y ello reluce particularmente en el hombre de modo que su singularidad constituye el centro de la antropologia cusana, de tal manera que la perfección consista en ser lo que es. Ello se plasma en la fórmula "Sé tú tu mismo y yo seré tuyo". El hombre no tiene que salir de sí para llegar a su plenitud, pues en la fidelidad absoluta a sí mismo descubrirá la plenitud de su ser. $Y$ este processo se realiza únicamente en el
ABSTRACT - Some reduced consideration of the medieval philosophy considers the synthesis achieved in the XIIIth century as the exclusive and completed pattern, so that, from that time forward, the later thinkers enter in a slope of decadence that will culminate with the rupture proclaimed by the modem thought. But the modern historiography allows us to evaluate the diversity of the proposals better that mark the $\mathrm{XIV}$ and XV centuries. One of these reasons is provided to us by the thought of Nicholas of Cusa (1401-1464) because in him it is evidenced how the alive history incorporates the past and project itself towar the future interpreting with originality its time. The present investigation seeks to intrude in one of the aspects maybe fewer explored of the cusan thinking: its ethical perspective. Nicholas of Cusa didn't write any specific treatise. However it bequeathed us a focus that we consider very rich. The starting point is born its conception of the singularity. The individual is not an isolated monad and desconected of the universe. For Nicholas of Cusa in each reality the maximum "contracto" is given. It is.a dense expression that expresses the presence of the infinite thing in the finite thing. Each entity that participates of the universe is also unrepeatable and unrepleaceable. And it glitters particularly in the man so that their singularity it constitutes the center of the cusan anthropology, in such a way that the perfection consists on being what is. It is captured it in the formula "Be you yourself and I will be yours." The man doesn't have to leave of itself to arrive to his fuliness, because in the absolute fidelity to itself will discover his being's fuliness. And this

* Universidad del Salvador, Buenos Aires. Gral. Artigas, 3170. 1417. Buenos Aires. Argentina.

\begin{tabular}{|l|l|l|l|l|l|}
\hline VERITAS & Porto Alegre & v. 44 & n. 3 & Setembro 1999 & p. 823-830 \\
\hline
\end{tabular}


ejercicio de la libertad que capacita o traba el acceso a la perfección moral, es decir, la plenitud del amor al que el hombre está destinado.

PALABRAS-CLAVE - Nicolás de Cusa. Ética. Individualidad. Retorno a Diós. process is only carried out in the exercise of the freedom that it qualifies or it locks the acess to the moral perfection, that is to say, the fuliness of the love to which the man is destined.

KEY WORDS - Nicolas of Cusa. Ethics. Individuality. Coming back to God.

Adentrarse en el pensamiento de Nicolás de Cusa es enfrentarse con la secular cuestión filosófica acerca de la relación de la unidad y la multiplicidad.

Desde sus primeros escritos preparados en el conflictivo clima que la situación político-jurídica de la Iglesia de su tiempo protagonizaba, propone la consigna liberadora de la "Concordantia Catholica" (Concordia universal) - tal es el título de la primera obra de envergadura (1433) - abogando decididamente en favor de la armonía generada a partir de la "representación" y de la "unidad", como único camino posible para resolver las enconadas divisiones que afectaban a los miembros de la Iglesia. Pero su tiempo, es decir el que muestra el "otoño de la edad media" - según la clásica expresión de J. Huizinga - y la gestión de la nueva visión humanístico-renacentista del "quattrocento", plantea a su inquieto espiritu arduas cuestiones científicas, filosóficas y teológicas. Ante este desafío Nicolás de Cusa no sólo reasume la sabiduría tradicional (prisca via) sino que no duda en asignar al hombre un rol central llamándolo no sólo "micro-cosmos", sino con audaz fórmula secundus deus.

Con incansable prolijidad elabora nuevos conceptos a partir de la docta ignorancia y los aplicará a todas las cuestiones metafísicas y antropológicas que investiga. Recoge de la aritmética y de la geometria el lenguaje y los símiles acerca del número, el punto, la línea, la esfera, el triángulo, ilustrando con ellos su pensamiento. Trabaja ahincadamente las nociones de máximo, de infinito, de mínimo, de igualdad, de conexión y de unidad pues la precisión y la posibilidad para pensarlos más allá de sus límites formales le permite acercarse mejor a su propósito de penetrar la realidad de lo infinito y lo finito. Y mediante los pares conceptuales de complicación - explicación, contracción - maximidad, precisión - conjetura, unidad - multiplicidad, propone clarificar los diversos aspectos de la relación entre lo uno y lo múltiple; lo infinito y lo creado. Todo ello ensamblado en una originalísima sintesis que da a su pensamiento, como reconocen eminentes estudiosos, la fisonomía de una propuesta que no sólo responde a los problemas de su época sino que es precursora del nuevo camino que se inicia en la modernidad.

De entre el copioso material que nos legara queremos abordar algunas consideraciones que, estimamos, nos han de permitir descubrir su particular visión de la ética.

Si bien no escribió ningún tratado específico de esta materia, sin embargo toda la orientación de su obra no puede menos de tener en cuenta la problemática propia de la ética - finalidad del hombre, sentido de la libertad - al referirse ampliamente a temas antropológicos. 
En este sentido queremos ceñirnos particularmente a dos aspectos que consideramos relevantes : su concepción acerca de la singularidad de toda creatura y especialmente el hombre, y la particular. gravitación de la libertad en su realización.

En primer lugar, el punto de partida en todas sus reflexiones no es otro sino la maximidad de lo infinito. No se trata de construir una noción redundante, sino marcar explícitamente que únicamente desde tal principalidad podrá comprenderse la multiplicidad, la diversidad, la alteridad. De donde se presenta una segunda instancia de reflexión: cómo entender la creaturalidad siendo así que para Nicolás de Cusa entre lo finito y lo infinito es imposible establecer alguna proporción. Pero el hecho de la realidad múltiple y limitada y el hombre que participa de manera relevante en ese universo no permite eludir el tema. De ahí que aplique toda la lucidez de su reflexión para intentar explicar la correcta vinculación de ambos polos: lo infinito y lo finito. Desde la metafísica cusana es claro que lo máximo e infinito no puede no estar presente en lo limitado y finito: todo el ser de lo finito proviene, $a b$-esse nos dice en D.I.II, cap. II., de lo infinito. Pero esta presencia no es absorción y por ello afirma con decidida energía la insustituible singularidad de cada realidad, de modo que la considera irrepetible. Esta afirmación reviste especial importancia cuando la aplicamos al hombre. Aquí nos encontraremos no sólo con la afirmación de la singularidad estricta sino que la libertad tendrá un rol decisivo.

Intentaremos por lo tanto profundizar estas temáticas cusanas a la luz de aquellos textos que nos parecen más significativos y clarificantes, reconociendo, sin embargo, que un estudio exhaustivo de los temas implicaría desarrollos mucho más amplios y profundos.

\section{La singularidad}

La pluralidad no es para Nicolás de Cusa la manifestación de multiplicidades diversas y desconexas entre si. Desde su perspectiva la comprensión de la pluralidad sólo se adquiere si la contemplamos desde su fuente: la unidad. En este sentido nuestro autor se encolumna en la larga tradición platónica, que se muestra tan diversa y tan rica durante la configuración del medioevo y que retiene como uno de sus principios fundamentales la precedencia de lo uno sobre lo múltiple. Y aquí nos encontramos con uno de los conceptos básicos: el concepto de "contracto", o "concreto" como lo denomina alguna vez. El término hace referencia a lo determinado, a lo delimitado, a lo circunscrito. Pero en ello se destaca un matiz especial: la realidad contracta no muestra sino una realidad de un orden superior, en cuanto éste ha descendido y se ha hecho presente, de un modo peculiar, en un orden o realidad inferior. La contracción marca, precisamente, este descenso. Lo máximo, el principio, lo descendente se da en la creatura, cuyos rasgos propios, respecto de lo máximo, no muestra sino la imperfección que le es inherente: corruptibilidad, divisibilidad, imperfección, diversidad, pluralidad, etc.. Precisamente en su libro II acerca de la Docta Ignorancia, destinado a explicitar la relación de lo máximo con el universo, nos dice muy explícitamente: "Por tanto, la creatura tiene de Dios el que sea una, separada, y conectada al universo y cuanto más una 
tanto más semejante a Dios. Empero el que su unidad está en la pluralidad, la distinción en la confusión, la conexión en la discordancia, no lo tiene de Dios, ni tampoco de una causa positiva, sino contingente" (lib. II, cap. II, $\mathrm{n}^{\circ} 99$ ).

En el universo o mundo, es decir en la totalidad de las cosas, lo infinito no puede no estar presente, por cuanto es fuente de todo ser. Para referirse a esta condición aplica el término "contracto": "el mundo - nos dice - o universo es lo máximo contracto y uno...; principio contracto y fin de todas las cosas; ente contracto; infinitud contracta, de modo que sea contractamente infinito" (D.I. lib.II, cap. IV, $\mathrm{n}^{\mathbf{0}}$ 113).

Nicolás de Cusa es muy consciente de la dificultad que pueden provocar sus afirmaciones. No en vano quien ardorosamente lo combatiera, J. Wenck en su libello De ignota litteratura, lo estigmatiza por estas expresiones considerándolo panteísta. La respuesta cusana insiste en no confundir Dios y creatura. "¿Quién puede al fin entender - nos dice al cabo de sus reflexiones - que Dios es la forma de ser ('forma essendi') y que sin embargo no está mezclado a la creatura?" (D.I. libr. II, cap. II, $\mathrm{n}^{\mathrm{O}}$ 102).

En última instancia aun cuando invoquemos el tradicional vocabulario de la participación no lograremos dilucidar lo que es el acto exclusivo de Dios: "¿Cómo de modo diverso una forma infinita sea participada en creaturas diversas, siendo así que el ser de la creatura no puede ser otro que la misma forma reflejándose, no recibida en algún otro positivamente, sino diversa contingentemente?" (D.I. libr. II, cap. II, $n^{\circ}$ 103). Por ello mismo Nicolás de Cusa abundará en fórmulas que destacan los aspectos negativos de la creatura: "Por lo tanto, la creatura, que es serque-viene-de no tiene todo aquello que es, o sea la corruptibilidad, la divisibilidad, la imperfección, la diversidad, la pluralidad y demás cosas similares, (recibido) de lo máximo, eterno, indivisible, perfectísimo, inseparable, uno, ni tampoco de una causa positiva" (D.I. lib. II, cap. II, no 99).

Pero, en la visión cusana, la creatura no se agota con estos aspectos negativos necesarios para marcar la dependencia. Con notable firmeza pondera la irrepetible singularidad de toda creatura.

Cada realidad existente es, en la visión cusana, única, irrepetible, irreductible a una entidad común. Y junto a ello aparece otra nota: tal singularidad no puede ser pensada como "un modo de Dios" manifestándose concretamente.

Abordando el universo plural reconoce, en primer lugar, la vigencia de las categońas lógicas clasificatorias de los entes: "Es necesario - nos dice - que todas las cosas se diferencien o por el género, o por la especie, o por el número... por lo cual todas las cosas se distinguen por grados de modo que ninguno coincida con el otro" (D.I. lib. III, cap. 1ำ, $\mathrm{n}^{\circ}$ 182).

La última afirmación se explicita apodícticamente: "No subsisten los géneros sino contractamente en las especies, ni las especies sino en los individuos quienes son los únicos que existen en acto" (D.I. libr. III, cap. 1ํㅡㄴ $\mathrm{n}^{\circ}$ 184). Por tanto si desde esta singularidad contemplamos el universo, la conclusión que se impone será: "De modo que nada haya en el universo que no goce de una cierta singularidad, que no puede encontrarse en ningún otro" (D.I. libr. III, cap. 1, nº 188). 
En un interesante desarrollo en su libro De Venatione Sapientiae (1462) pasa de la abstracta reflexión matemática acerca del uno y del número, al "prado de la singularidad", como lo denomina allí. Reitera, en primer lugar, la in-multiplicabilidad de lo singular: "Lo singular abraza todas las cosas, pues todas son singulares y cada cosa inmultiplicable" (cap. XXI, $\mathrm{n}^{\circ}$ 65) y relaciona luego la singularidad de la creatura con la singularidad per essentiam que sólo compete a Dios y de la cual todos participan: "De donde como Dios es singularísimo y máximamente inmultiplicable, así después de Dios la singularidad del mundo es máximamente inmultiplicable y luego la de las especies, después la de los individuos, de los cuales ninguno es multiplicable. Pues en esto todas las cosas se alegran en participar la semejanza de Dios" (ibid., $\mathrm{n}^{\circ}$ 65). Afirmar, por otra parte, tal origen divino es asegurar la perdurabilidad de lo singular: "Lo singular, puesto que ha sido singularizado por la causa eterna, jamás podrá disolverse en lo no singular" (ibid., no 66). Es en el marco de las afirmaciones sobre la singularidad donde nos encontramos con una aplicación plena de plasticidad y no exenta de humor. Se trata de la consideración que propone a su amigo el Cardenal Julián Cesarini, a quien dedicara sus obras Sobre la Docta Ignorancia y Sobre las Conjeturas. Nos encontramos en el segundo libro Sobre las Conjeturas a propósito de las diferencias y las concordancias con este párrafo: "La singularidad, por tanto, todo lo singulariza, la especificación todo lo especifica, la generalización todo lo generaliza, la universalidad lo universaliza. Pues todo lo universal, lo general y lo especifico en Julián son Julián ('iulanizant' dice creando un neologismo), tal como la armonía en el laúd es laúd y en la cítara cítara. Ni tampoco esto es posible en otro como en ti. Ahora bien, esto que es en ti Julián, es el ser Julián ('est iulanizare'), en todos los hombres, es ser hombre ('humanizare'), en los animales, ser animal ('animalizare') y así en los demás" (De Coniecturis, lib. II, cap. III, nํ 89). Sin embargo la acentuada afirmación de la singularidad no desconoce otro aspecto imprescindible en la visión metafísica cusana: la relación de esta singularidad con su fuente:

"Por tanto la singularidad incorruptible es la que formaliza y conserva todas las cosas y todas las cosas con un deseo plenamente natural tienden a la causa de su singularidad en cuanto bien suficiente y perfecto de todas sus singularidades" (De Venatione Sapientiae, cap. XXII, nº 67).

$Y$ es el marco de este retorno aquél en el que se inscriben minuciosamente los pasos que Nicolás de Cusa describe para que el hombre alcance su plenitud con el ejercicio de su libertad.

\section{El camino hacia la visión de Dios}

En el texto Acerca de la visión de Dios (1453) dedicado a los monjes de Tegernsee y escrito con ocasión de las controversias que se agitaban, en ese tiempo, acerca de la esencia de la mística, Nicolás de Cusa nos expone no sólo su sentencia sobre el carácter cognoscitivo de la experiencia mística, sino que advertimos la profunda visión del hombre, su destino y su libertad.

El texto adopta un lenguaje coloquial entre el hombre y Dios característico en este género de obras, y revela con este procedimiento la profundidad del compromiso personal que implicaban las consideraciones que desarrolla. "Tu ser, Señor, 
no abandona mi ser; pues en tanto yo soy, en cuanto tú eres conmigo. Y puesto que tu ver es tu ser, por ello yo soy, porque tú me miras, y si sustrajeras tu rostro de mí, de ninguna manera subsistiría" (De Visione Dei, cap. IV, n 20). La metáfora de la mirada implica no sólo el conocimiento perfecto de parte de Dios, sino que alude al tema central: la providencia o presencia amorosa que procura a cada creatura el ser de la mejor manera posible: "De tal manera, Señor, miras tú cada cosa que es, que no puede concebirse para todo aquello que es que tu cuidado no es otro sino que ello sea tan sólo del modo mejor que puede ser" (ibid. nº 9).

"Tu verme es el ser visto por mí - resume Nicolás esta dialéctica del ser visto y del ver a Dios - viéndome a mí haces que tú seas visto por mí, y no otra cosa es verte que el que tú veas a quien te ve" (De Visione Dei, cap. V, ํㅜ 15). El ver se atribuye no sólo a Dios. Si así fuera podría pensarse en una actitud meramente receptiva de parte del hombre. Para éste, el ver es el resultado de ser visto. Dios viendo me hace que lo vea y ver a Dios no es otra cosa que Dios me vea en cuanto vidente de Él. Formulación evidentemente audaz, pero que expresa el sublime grado de intimidad y de reciprocidad de la experiencia mística. Sin embargo, Nicolás es consciente de la infinita distancia entre Dios y el hombre. Por un lado la inaccesible grandeza y por otro la insuficiencia humana. Y estando en el silencio de estas reflexiones, nos dice, Dios respondió en lo más íntimo del corazón: "Sé tú mismo y yo seré tuyo" (sis tu tuus et ego ero tuus) (De Visione Dei, cap. VII, n⿳o 25).

Llama, en primer lugar, poderosamente la atención que, en este momento álgido del acceso a Dios, la expresión cusana asiente la experiencia en el retorno a sí mismo, asegurando que es en la fidelidad a nosotros mismos, como Dios se hará nuestro. Además, a continuación de estas expresiones Nicolás nos dice que todo está pendiente del uso que hagamos de la libertad. Que el hombre quiera ser lo que es, está sometido a su propia libertad y el mismo Dios condicionó a esta decisión el ser posesión del hombre.

"Oh, Señor - exclama a continuación - suavidad de toda dulzura, has puesto en mi libertad que yo sea, si lo quisiere, yo mismo; de aquí que si yo no fuera yo mismo, tú no serías mío, porque coaccionarías la libertad que tú no pudieras ser mío, si también yo no fuera yo mismo. Y como ello está en mi libertad, no me obligas, sino que esperas que yo elija ser yo mismo" (De Visione Dei, cap. VII, nº 27).

Por tanto esta cima de perfección que sólo se adquiere con el ejercicio de la libertad, requiere una docencia. El camino propuesto es el del orden, en el que el sentido obedece a la razón y donde la razón domina guiada por el Verbo que es razón de todas las razones. "¿Cómo seré yo mismo - se pregunta el autor - si tú, Señor, no me lo enseñas? Esto es empero lo que me enseñas: que el sentido obedezca a la razón y la razón domine. Por tanto cuando el sentido sirve a la razón soy yo mismo, pero la razón no tiene de dónde ser guiada sino por ti, Señor, que eres el Verbo y la razón de las razones" (ibid. $n^{0} 28$ ). Los capítulos subsiguientes habrán de elaborar con amplitud el significado de este tornar a sí mismo para llegar a participar de la visión de Dios. 
Pero, desde nuestro punto de vista, queremos señalar el significado antropológico que descubrimos en esta fórmula. $Y$ en primer lugar advertimos que tal recomendación a ser uno mismo se funda en la concepción de singularidad a la que nos hemos referido. Ser plenamente lo que se es, es asumir, conforme a la perspectiva cusana, la concreta entidad con la conciencia de su singularidad irrepetible. Sólo quien tiene la mirada preparada para descubrir lo máximo contracto, como se esfuerza en mostrarlo Nicolás de Cusa, descubre la riqueza verdaderamente infinita que lo singular en cuanto tal conlleva.

No es necesario salir de sí, y volcarse a una acción múltiple; tampoco es necesario buscar el fundamento en conceptos generales. Lo decisivo es que lo infinito está presente en lo finito y ello es lo que revela la legítima riqueza del hombre.

Pero la singularidad, por otra parte, está sujeta a una decisión. El ejercicio de la libertad hará que la asumamos o la frustremos. Dios mismo aguarda, sin coaccionar: "Sis tu tuus". Decídete, elige, sé lo que tú eres; advierte que la intimidad de tu ser está alimentada por la fuente del ser. Dios, repite constantemente Nicolás de Cusa recuperando la tradición del Maestro Eckhart y de la Escuela de Chartres, es la forma essendi, la forma de ser de la creatura. Por lo tanto, este ejercicio de la libertad debe tener en cuenta una ontología: si el hombre renuncia a ser lo que es, estará negándose su propio ser.

Pensamos que aquí se encuentra el punto de partida de la propuesta ética cusana: la ética surge, primariamente, del interior, desde lo que el hombre es. Y sólo el ejercicio responsable de la libertad en cuanto fidelidad a sí mismo, hará que el hombre descubra su auténtico ser, el cual es también maximidad contracta en la singularidad irrepetible de su persona.

\section{Nota bibliográfica}

\section{Fuentes: obras de Nicolás de Cusa*}

De Docta Ignorantia I, Schriften des Nikolaus von Kues in deutscher Übersetzung. Im Auftrag der Heidelberger Akademie der Wissenschaften, hrsg. von E.Hoffmann +, Paul Wilpert + und Karl Bormann. Heft. 15a. Lateinisch-deutsche Parallelausgabe Felix Meiner Verlag. Hamburg 1979.

De Docta Ignorantia II, Idem. hrsg. von Paul Wilpert + . Zweite verbesserte Auflage besorgt von Hans Gerhard Senger, Lateinisch-Deutsch. Felix Meiner Verlag. Hamburg 1977.

De Docta Ignorantia III, Idem. hrsg. von Hans Gerhard Senger. Lateinisch-Deutsch. Felix Meiner Verlag, Hamburg 1977.

De Coniecturis, Schriften des Nikolaus von Kues in deutsche Übersetzung. In Auftrag der Heildelberger Akademie der Wissenschaften hrsg.von Ernst Hoffmann+, Paul Wilpert+ u. Karl Bormann Heft. 17. Lateinisch-deutsche Parallelausgabe. Felix Meiner Verlag. Hamburg 1988.

De Venatione Sapientiae, Nicolai de Cusa, Opera Omnia iussu et auctoritate Academiae Litteranum Heildelbergensis. XII. Ediderunt Raymundus Klibansky et Johannes Gerhardus Senger. Hamburg: in Aedibus Felicis Meiner. MCMLXXXII.

De Visione Dei, Nikolaus von Kues Werke (Neuausgabe des Strassburger Drucks von 1488) Band II. Hrsg. P.Wilpert + Walter de Gruyter Verlag / Berlin 1967.

* En todos los casos las traducciones del texto latino son responsabilidad del autor. 


\section{Estudios sobre el pensamiento cusano}

Giovanni Santinello: Introduzione a Niccoló Cusano. Laterza. Bari. 1987. Completísima introducción no sólo por su claridad para exponer el pensamiento cusano, sino también por el repertorio bibliográfico actualizado y una enjundiosa Storia della critica que describe el itinerario de las interpretaciones cusanas.

Klaus Kremer: "Gottes Vorsehung und die menschliche Freiheit" (sis tu tuus, et ego ero tuus). En Das Sehen Gottes nach $N$. von Kues, Mitteilungen und Forschungsbeiträge der Cusanus- Geseelschaft. (MFCG). N² 18, 1989.

Heimsoeth, Heinz: Los seis grandes temas de la Metafísica Occidental. Cap. I y II.

Revista de Occidente. Madrid. 1959. 